\title{
Transient receptor potential vanilloid 4 promotes the growth of non-small cell lung cancer by regulating Foxp3
}

\author{
Jiang-tao Pu凶, Tao Zhang, Kai-ming He, Deng-guo Zhang, Zhang-yu Teng and Yun-fei Wu \\ Department of Thoracic Surgery, The Affiliated Hospital of Southwest Medical University, Luzhou, Sichuan, 646000 China
}

\begin{abstract}
Transient receptor potential vanilloid 4 (TRPV4) functions in malignant tumours. However, the role of TRPV4 in non-small cell lung cancer (NSCLC) remains unclear. In this study, we demonstrated that TRPV4 expression was upregulated in NSCLC tissues and cell lines. The TRPV4 levels in NSCLC patients and cell lines were detected, and the function of TRPV4 was studied both in vivo and in vitro. The level of TRPV4 showed a positive correlation with the tumour size in NSCLC patients. Activation of TRPV4 by the agonist GSK1016790A promoted cell proliferation and decreased apoptosis in A549 cells, and these effects were enhanced when the cells overexpressed TRPV4. Moreover, the inhibitory effects of GSK1016790A on the apoptosis of A549 cells were impaired when GSK1016790A was used together with the TRPV4 selective antagonist HC- 067047 or when the cells were transfected with TRPV4 siRNA to downregulate TRPV4 expression. In an in vivo study, pharmacological inhibition of TRPV4 prevented A549 cell-derived transplanted tumour growth. The Foxp3 levels were significantly increased in NSCLC tissues and showed a positive correlation with the TRPV4 levels. Inhibition of TRPV4 using TRPV4 siRNA or HC-067047 significantly reduced the expression of Foxp3 in GSK1016790A-treated NSCLC cells. Moreover, downregulation of Foxp3 by transfection with Foxp3 siRNA significantly impaired TRPV4-induced NSCLC cell proliferation in vitro. The antitumour effects caused by TRPV4 inhibition in NSCLC might be attributed to the suppression of Foxp3, which subsequently induced cell apoptosis. Thus, pharmacological inhibition of TRPV4 may be a promising option for NSCLC treatment.
\end{abstract}

Keywords: Apoptosis; Foxp3; Non-small cell lung cancer; TRPV4

Received: 15 February, 2021; revised: 02 April, 2021; accepted: 14 April, 2021; available on-line: 07 January, 2022

凶e-mail: pujiangtao1972@sina.com

Acknowledgements of Financial Support: This work was financially supported by the Sichuan University Education Foundation (TSCI002).

Abbreviations: HRP, horseradish peroxidase; NSCLC, non-small cell lung cancer; TRPV4, transient receptor potential vanilloid 4

\section{INTRODUCTION}

Lung cancer is the most prevalent malignancy and leading cause of cancer-associated deaths around the world. Approximately $85 \%$ of lung cancer patients have non-small cell lung cancer (NSCLC), which is a group of histological lung cancer subtypes (Siegel et al., 2017). In North America, 154,050 Americans were expected to die from lung cancer in 2018, and this a rate exceeds the total numbers of deaths due to colon, breast, and prostate cancers. In eastern Asia, for example China, lung can- cer incidence peaked during the past 2 decades (Siegel et al., 2017; Chen et al., 2016). Many new insights into the causes and pathophysiological mechanisms of NSCLC have been reported in the past few decades, and some novel effective treatments are already available in the clinic to treat NSCLC; however, the five-year survival rate of NSCLC patients remains lower than 15\% (Luo et al., 2017). Undoubtedly, it is of critical importance to explore novel therapeutic agents for the treatment of this lethal malignancy.

Transient receptor potential (TRP) channels are a large family of ion channels that nonsel5ectively transport cations, such as sodium, calcium and magnesium, across the plasma membrane. There are several subgroups of TRP channels, including TRPC (canonical), TRPV (vanilloid), TRPM (melastatin), TRPML (mucolipin), and TRPP (polycystin) (Luo et al., 2017). Among these subgroups, TRPV has been extensively studied due to its biological role in affecting a variety of physiological and pathological processes. TRPV has been shown to participate in nociception development, heat sensation, inflammation and $\mathrm{Ca}^{2+}$ reabsorption in kidneys and bones (Santoni \& Farfariello, 2011). TRPV4 is a member of the TRPV family and has recently been found to regulate tumour cell proliferation, apoptosis, angiogenesis, migration and invasion during tumour progression (Santoni et al., 2011). In recent investigations (Ou-Yang et al., 2018; Wang et al., 2020; Huang et al., 2019; Fang et al., 2018; Liu et al., 2019), TRPV4 was reported to be involved in the progression of several types of malignant tumours, such as glioma, gastric cancer, oesophageal squamous cell carcinoma, hepatocellular carcinoma and colon cancer. Based on universal changes in TRPV4 expression (either increased or decreased mRNA and protein expression) and tumour-related bioactivity, TRPV4 has been proposed to be a promising diagnostic or prognostic marker as well as a therapeutic target for pharmaceutical intervention. Therefore, more studies are required to increase our knowledge of the types of neoplasms in which TRPV4 plays a role as well as the underlying molecular mechanisms. In this study, we identified the characteristics of TRPV4 and the molecular mechanism by which it participates in the development of NSCLC.

\section{MATERIALS AND METHODS}

\section{Patient specimens}

Tumour tissues and para-cancerous tissues were collected from NSCLC patients (62 patients) who underwent surgery at the Affiliated Hospital of Southwest Medical University of China (Luzhou, China) from 
April 2018 to May 2019. None of the patients received preoperative tumour treatments, such as chemoradiotherapy and biological immunotherapy. The histological types of the tumours were determined according to the 2015 WHO classification (Travis et al., 2015). The current study was approved by the Ethics Review Board of the Affiliated Hospital of Southwest Medical University (Z2018001) and was conducted in accordance with the ethical standards of the committee responsible for human experimentation (at both the institutional and national levels) and in accordance with the Declaration of Helsinki of 1975, as revised in 2000. All the patients signed informed consent forms.

\section{Immunohistochemical staining}

The expression of TRPV4 in the tumour and paracancerous tissues was determined by semiquantitative immunohistochemical analysis. Briefly, the tissues were embedded in paraffin and sectioned. The sections $(5 \mu \mathrm{m})$ were deparaffinized in xylene, hydrated with different concentrations of ethanol and blocked with $5 \%$ bovine serum albumin. Immunostaining was performed by incubation of the sections with s primary antibody (TRPV4 or Foxp3, Santa Cruz, CA, USA) at $4^{\circ} \mathrm{C}$ overnight and incubation with a horseradish peroxidase (HRP)-labelled secondary antibody at room temperature for $1 \mathrm{~h}$. Finally, the sections were incubated with diaminobenzidine substrate (Sigma, St. Louis, MO, USA). The immunostaining results were evaluated with light microscopy (Olympus) at $200 \times$ magnification.

\section{Cell culture}

The human NSCLC cell lines A549, H1299, and H226 and the normal lung epithelial cells BEAS-2B were purchased from the American Type Culture Collection (Manassas, VA, USA). All the cell lines were cultured in RPMI 1640 medium containing 10\% foetal bovine serum (HyClone, USA) at $37^{\circ} \mathrm{C}$ in a humidified atmosphere of $5 \% \mathrm{CO}_{2}$.

\section{Cell transfection}

TRPV4 (or Foxp3) was knocked down in cells using TRPV4 (or Foxp3) siRNA constructed by GenePharma (Shanghai, China). The following sequences were used: TRPV4 siRNA: 5'-GGAGCTGAACAAGAACTCA-3'; Foxp3 siRNA: 5'-GGCGGACCAUCUUCUGGAU-3'. Nonsilencing siRNA (5'-TTCTCCGAACGTGTCACG-3') was used as the negative control. Moreover, to overexpress TRPV4, a full-length fragment of TRPV4 cDNA was cloned into pcDNA 3.1. Transfection was conducted using Lipofectamine 2000 ${ }^{\mathrm{TM}}$ (Invitrogen), and the silencing efficiency was determined by qRT-PCR.

\section{Cell viability}

The cells were cultured in 96-well plates (Corning, Acton, MA, USA) at a density of 8000 cells per well and were treated with GSK1016790A (100 nM, Sigma, St. Louis, MO, USA) in the presence/absence of the TRPV4 antagonist HC-067047 (1 $\mu \mathrm{M}$; Sigma, St. Louis, MO, USA) for different time points $(24-72 \mathrm{~h})$. To detect cell viability, the cells were incubated with $10 \mu \mathrm{l} /$ well Cell Counting Kit-8 solution (Sigma, St. Louis, MO, USA). Subsequently, the cells were incubated for an additional $4 \mathrm{~h}$, and the optical density of each well was measured at $450 \mathrm{~nm}$ by using a microplate reader (Thermo Scientific, MA, USA).

\section{Apoptosis analysis}

A549 cells were cultured until they reached 90\% confluence, and then, the cells were cultured for another $48 \mathrm{~h}$. Apoptosis was assessed by flow cytometry analysis according to the manufacturer's instructions. Briefly, all the cells were harvested and stained with $5 \mu \mathrm{l} \mathrm{An-}$ nexin V-FITC and $5 \mu \mathrm{l}$ PI (BD Biosciences, San Jose, CA, USA). Apoptosis quantification was conducted with a FACSCalibur flow cytometer (Becton-Dickinson, San Jose, CA, USA). To assess apoptosis in vivo, all the specimens were fixed in $4 \%$ paraformaldehyde overnight at $4^{\circ} \mathrm{C}$, permeabilized with $0.1 \%$ Triton $\mathrm{X}-100$ and then incubated with TUNEL staining solution (Beyotime, Haimen, China) for $1 \mathrm{~h}$. The TUNEL-positive cells in five randomly selected fields of the slide were counted under a microscope (Olympus).

\section{Quantitative RT-PCR}

Total RNA was extracted from tumour tissues using TRIzol (Invitrogen, CA, USA) according to the manufacturer's instructions. Fold changes in mRNA expression were calculated by the relative quantification method $\left(2^{-\Delta \Delta C t}\right)$. The primers used in this current study were as follows:

TRPV4 forward: 5'-TCACTCTCACCGCCTACTACCA-3'; reverse: 5'-CCCAGTGAAGAGCGTAATGA

CC-3'; Foxp3 forward: 5'-GTGGCCCGGATGTGAGAAG-3'; reverse: 5'-GGAGCCCT'TGTCGG

ATGATG-3'; GAPDH forward: 5'-AGCCACATCGCTCAGACAC-3'; and reverse: 5'-GAAGGTGAAGGTCGGAGTC-3'.

GAPDH was used as the endogenous control.

\section{Western blot analysis}

Cell extracts were prepared using lysis buffer (Cell Signaling Technology, Beverly, MA, USA). Moreover, tissues were lysed with RIPA buffer (Sigma, St. Louis, $\mathrm{MO}, \mathrm{USA}$ ) to harvest the proteins. A BCA protein assay kit (Beyotime, Shanghai, China) was used to measure the concentrations of the proteins. Equal amounts of protein (50 $\mu \mathrm{g}$ per sample) were loaded and separated by SDSPAGE. After electrophoresis, the proteins in the SDSPAGE gel were transferred onto a polyvinylidene fluoride membrane. The membrane was incubated with primary antibodies overnight at $4^{\circ} \mathrm{C}$. After incubation, the membrane was further incubated with HRP-conjugated secondary antibodies for $1 \mathrm{~h}$. Finally, the proteins were detected and visualized using enhanced chemiluminescence (Tiangen Biotech Co., Ltd., Beijing, China). The band densities were measured by ImageJ software $\mathrm{NIH}$, Bethesda, MD, USA) and normalized to that of GAP$\mathrm{DH}$. The following antibodies were used: anti-TRPV4 and Foxp3 (Santa Cruz Biotechnology, Inc., CA, USA).

\section{Tumour formation assay}

The animal studies were approved by the Animal Care and Use Committee of Southwest Medical University (SL20170874) and conducted in accordance with the U.K. Animals (Scientific Procedures) Act, 1986, and the associated guidelines. A total of twenty male athymic BALB/c nude mice (5-6 weeks old) were purchased from the Model Animal Research Center of Nanjing University (Nanjing, China). The animals were housed in a temperature-controlled room with a 12-h light/12$\mathrm{h}$ dark cycle and were provided with autoclaved chow and water ad libitum. To establish a xenograft animal model, each mouse received subcutaneous injection 
of $2 \times 10^{6}$ A549 cells at a single site on the side of the posterior flank. When the tumour size reached approximately $100 \mathrm{~mm}^{3}$, all the mice were randomized into the following groups and received the indicated intraperitoneal injection treatment daily: control group, HC067047 (10 mg/kg) group, GSK1016790A (10 $\mu \mathrm{g} / \mathrm{kg})$ group, and GSK1016790A (10 $\mu \mathrm{g} / \mathrm{kg})+\mathrm{HC}-067047$ (10 $\mathrm{mg} / \mathrm{kg} /$ day) group. The animals were sacrificed 40 days after injection, and the tumour tissues were removed and weighed.

\section{Statistical analysis}

All the values are presented as the means and standard deviations. The statistical analyses were performed using the SPSS statistical software package standard version 17.0 (IBM, Inc., Chicago, IL, USA) Comparisons between multiple groups were assessed via one-way analysis of variance followed by LSD test. Chi-square test was used for classification data. Correlation analyses were performed using GraphPad Prism version 8.0 (GraphPad Software, San Diego, CA, USA).

\section{RESULTS}

\section{TRPV4 expression was positively correlated with the development of NSCLC in patients}

To examine the role of TRPV4 in the development of NSCLC, we detected the level of TRPV4 in cancer tissues from patients. As shown in Fig. 1, the expression of TRPV4 was evaluated by western blotting and immunohistochemical staining, and the results showed that the level of TRPV4 was significantly higher in tumour tissues than in paired para-cancerous tissues $(P<0.01)$. Moreover, we further attempted to analyse the relationship between TRPV4 and the clinical parameters of the NSCLC patients. To this end, we divided the patients into high and low expression groups based on the median TRPV4 expression level. As shown in Table 1,

Table 1. Relationship of TRPV4 expression with the pathological features of NSCLC patients

\begin{tabular}{|c|c|c|c|c|}
\hline \multirow{2}{*}{ Clinicopathological features } & \multirow{2}{*}{ Number } & \multicolumn{2}{|c|}{ TRPV4 expression } & \multirow{2}{*}{$P$ value } \\
\hline & & Low $(n=31)$ & High $(n=31)$ & \\
\hline \multicolumn{5}{|l|}{ Ages (years) } \\
\hline$\geq 60$ & 37 & 15 & 22 & 0.120 \\
\hline$<60$ & 25 & 16 & 9 & \\
\hline \multicolumn{5}{|l|}{ Sex } \\
\hline Male & 30 & 18 & 12 & 0.204 \\
\hline Female & 32 & 13 & 19 & \\
\hline \multicolumn{5}{|l|}{ Tumour size } \\
\hline$\geq 3 \mathrm{~cm}$ & 36 & 11 & 25 & 0.001 \\
\hline$<3 \mathrm{~cm}$ & 26 & 20 & 6 & \\
\hline \multicolumn{5}{|l|}{ TNM stage } \\
\hline$|-| \mid$ & 28 & 12 & 16 & 0.444 \\
\hline III-IV & 34 & 19 & 15 & \\
\hline \multicolumn{5}{|l|}{ Differentiation } \\
\hline Good/Moderate & 30 & 20 & 14 & 0.202 \\
\hline Poor & 32 & 11 & 17 & \\
\hline
\end{tabular}

A total of 62 patients were divided into the high and low TRPV4 expression groups based on the median TRPV4 expression level.
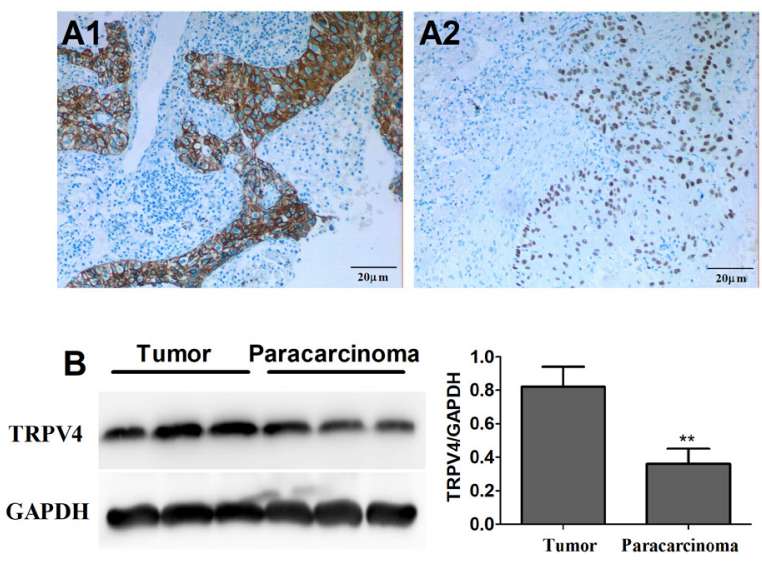

Figure 1. TRPV4 expression in specimens from NSCLC patients. (A) TRPV4 expression in tumour tissues (A1) and paracarcinoma tissues (A2) from NSCLC patients was detected by immunohistochemical staining. (B) TRPV4 expression in tumour and paracarcinoma tissues was detected by western blotting. The data are presented as the means \pm SDs. ${ }^{* *} P<0.01$ vs the tumour $(n=62)$.

TRPV4 was found to be negatively correlated with tumour size $(P<0.01)$.

\section{TRPV4 promoted the proliferation of NSCLC cells in vitro}

As shown in Fig. 2A, we further examined TRPV4 expression in human NSCLC cell lines and found that TRPV4 expression was significantly higher in all the human NSCLC cell lines than in the normal lung epithelial cell line BEAS-2B $(P<0.01)$. Briefly, a high level of TRPV4 was observed in the H226 cells, while a relatively low level of TRPV4 was observed in the A549 cells. To explore the biological function of TRPV4 in the proliferation of NSCLC cells, we downregulated and overexpressed TRPV4 in A549 NSCLC cells by transfection of TRPV4 siRNA and a TRPV4 overexpression vector, respectively. As shown in Fig. 2B-2C, the 

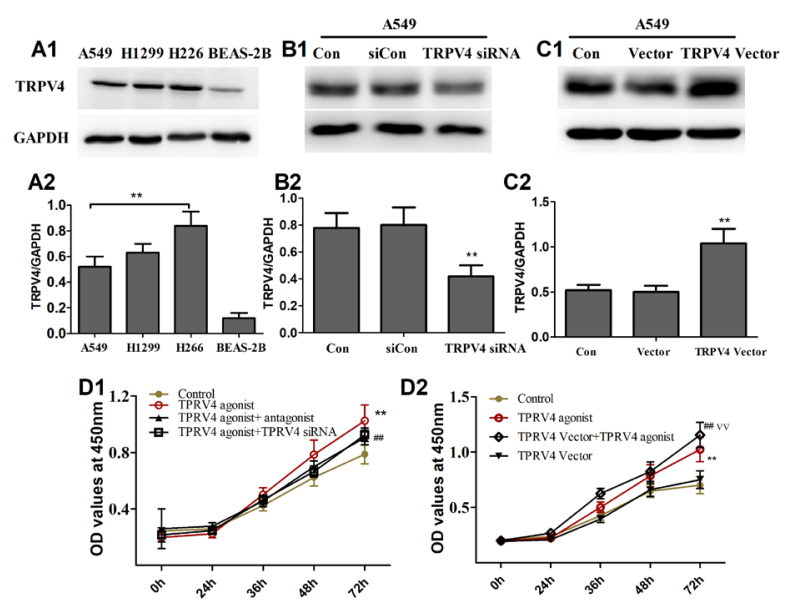

Figure 2. Activation of TRPV4 promotes NSCLC cell proliferation. (A1-A2) TRPV4 expression was detected in the NSCLC cell lines A549, H1299, H226 and in the normal lung epithelial cell line BEAS-2B by western blotting. ${ }^{* *} P<0.01$ vs the BEAS-2B cells. (B1B2) TRPV4 was knocked down in A549 cells by transfection with TRPV4 siRNA. ${ }^{* *} P<0.01$ vs Con or siCon. (C1-C2) TRPV4 was overexpressed in A549 cells by transfection of the TRPV4 vector. ${ }^{* *} P<0.01$ vs the Con or vector. (D1) A549 cells treated with $1 \mu \mathrm{M} \mathrm{HC}-067047$ or TRPV4 siRNA in the presence of 100 nM GSK1016790A, and then, the viability of the cells was determined by CCK- 8 assay. ${ }^{* *} P<0.01$ vs the Control; ${ }^{\#} P<0.01$ vs the TRPV4 agonist; (D2) A549 cells transfected with the TRPV4 vector and/or treated with the TRPV4 agonist GSK1016790A (100 nM), and then, the viability of the cells was determined by CCK- 8 assay. ${ }^{* *} P<0.01$ vs the Control; ${ }^{\#} P<0.01$ vs the TRPV4 agonist; $\nabla \nabla P<0.01$ vs the TRPV4 vector. The data are presented as the means \pm S.D.s of three parallel measurements.

TRPV4 levels were decreased by approximately 50\% and increased by nearly 2.4-fold in the A549 cells, respectively $(P<0.01)$. As shown in Fig. 2D, overexpression of TRPV4 markedly promoted cell proliferation, while downregulation of TRPV4 significantly decreased the viability of NSCLC cells in the presence of the TRPV4 agonist GSK1016790A $(P<0.01)$. Moreover, treatment with GSK1016790A or the TRPV4 antagonist HC-067047 significantly promoted or inhibited the proliferation of NSCLC cells, respectively. Our findings demonstrate that TRPV4 activation enhances the proliferation of NSCLC cells.

\section{TRPV4 inhibited the apoptosis of NSCLC cells in vitro}

As shown in Fig. 3, compared with the control treatment, treatment with GSK1016790A decreased apoptosis $(P<0.01)$. However, overexpression of TRPV4 in the A549 cells further enhanced the inhibitory effects of GSK1016790A on apoptotic death $(P<0.01)$. Therefore, it was suggested that TRPV4 negatively regulates the apoptosis of NSCLC cells.

\section{TRPV4 upregulated Foxp3 in NSCLC}

It has been previously reported that Foxp3 facilitates the formation of NSCLC and promotes its growth and metastasis (Yang et al., 2017). To better understand the molecular mechanism associated with the ability of TRPV4 to promote NSCLC, we hypothesized that TRPV4 may have a relationship with Foxp3. As shown in Fig. 4A, Foxp3 expression was significantly higher in tumour tissues than in paired para-cancerous tissues $(P<0.01)$. Furthermore, the level of TRPV4 was positively correlated with the expression of Foxp3 in the tumour tissues from NSCLC patients (Fig. 4B; Spearman
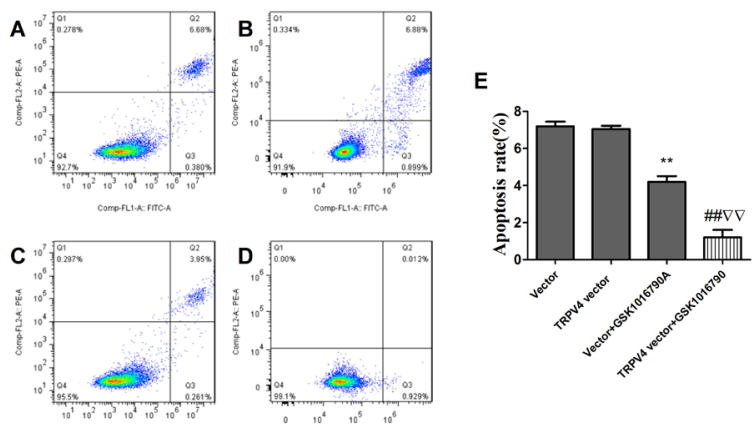

Figure 3. Activation of TRPV4 decreases apoptotic death in NSCLC cells.

Annexin V-FITC/ PI double staining was used to assess the apoptosis of A549 cells. (A) Cells treated with vector control; (B) Cells treated with TRPV4 vector; (C) A549 cells treated with GSK1016790A (100 nM); (D) A549 cells treated with TRPV4 vector and GSK1016790A (100 nM); (E) Apoptosis was analysed by a FACSCalibur flow cytometer. The data are shown as the means \pm SDs and are representative of three independent experiments. ${ }^{*} P<0.01$ compared with vector; ${ }^{\# \# P}<0.01$ vs GSK1016790A; $\nabla \nabla P<0.01$ vs TRPV4 vector.
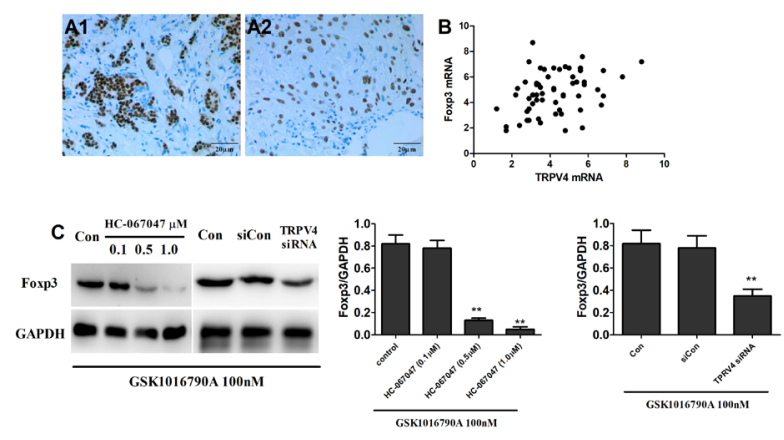

Figure 4. Foxp3 is upregulated in NSCLC patients.

(A) Expression of Foxp3 in tumour tissues (A1) and paracancerous tissues (A2) of NSCLC patients was measured by immunohistochemical staining. (B) Correlation analysis of TRPV4 and Foxp3 expression $(n=62)$. mRNA levels of TRPV4 and Foxp3 were detected by qRT-PCR. (C) A549 cells were treated with HC-067047 at different concentrations for $48 \mathrm{~h}$ or transfected with TRPV4 siRNA for $48 \mathrm{~h}$ in the presence of $100 \mathrm{nM}$ GSK1016790A. Then, the intracellular proteins were extracted and quantitatively analysed by western blotting. The data are presented as the means \pm S.D.s of three measurements. ${ }^{* *} P<0.01$ compared with the control.

$r=0.2916$; $P=0.0215)$. Consistent with these results, further study showed that treatment with TRPV4 siRNA or TRPV4 antagonist significantly reduced the expression of Foxp3 in NSCLC cells in the presence of the TRPV4 agonist GSK1016790A (Fig. 4C; $P<0.01$ ), suggesting a positive regulatory effect of TRPV4 on the expression of Foxp3.

\section{Downregulation of Foxp3 impaired TRPV4-induced NSCLC cell proliferation in vitro}

Next, we investigated whether Foxp3 could affect the TRPV4-mediated promotion of growth. First, we treated A549 cells with agonists and then observed their response to transfection with Foxp3 siRNA. As shown in Fig. 5A, Foxp3 siRNA successfully knocked down Foxp3 expression in the A549 cells $(P<0.01)$. As shown in Fig. 5B-5C, GSK1016790A promoted the proliferation and prevented the apoptotic death of the A549 cells $(P<0.01)$. Moreover, it was interesting to observe that knockdown of Foxp3 impaired the TRPV4 agonist- 


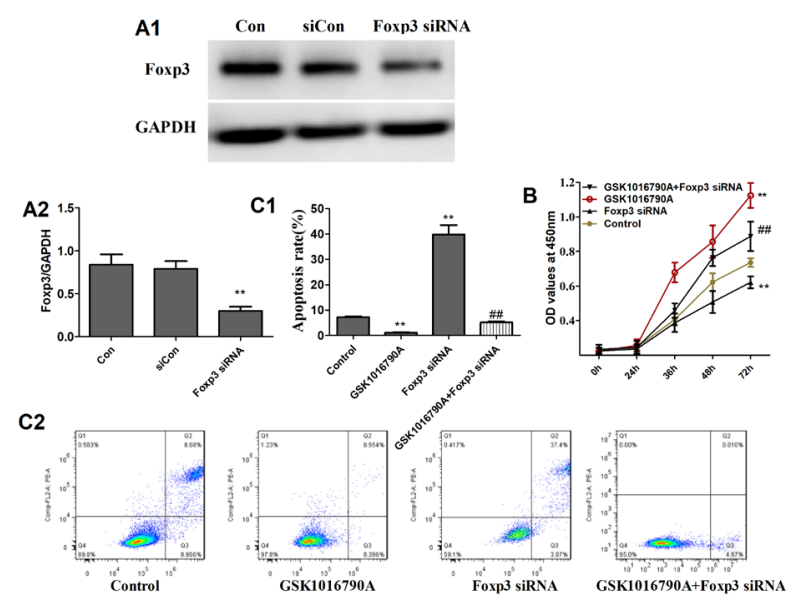

Figure 5. Downregulation of Foxp3 impaired TRPV4-mediated NSCLC cell proliferation and apoptosis in vitro.

(A1-A2) Foxp3 was knocked down in A549 cells by transfection with Foxp3 siRNA, and the effects of Foxp3 knockdown were assessed by western blotting. (B) A549 cells were transfected with Foxp3 siRNA or treated with the TRPV4 agonist GSK1016790A $(100 \mathrm{nM})$ for $48 \mathrm{~h}$, and then, the viability of cells was determined by CCK-8 assay. (C) Apoptosis in different groups was measured by flow cytometry analysis. ${ }^{* *} P<0.01$ vs the Control (Con). ${ }^{\# \#} P<0.01$ vs the GSK1016790A group. The data are presented as the means \pm S.D.s of three parallel measurements.

mediated proliferation and apoptosis of the A549 cells $(P<0.01)$.

\section{Inhibition of TRPV4 prevented the development of NSCLC in vivo}

Finally, we verified the positive effects of TRPV4 on the growth of NSCLC in vivo. First, we established mice with A549 cell-derived transplanted tumours, and the tumour growth was monitored and the weight was measured at the end of study. As shown in Fig. 6A-6B, tumour growth was significantly enhanced by treatment
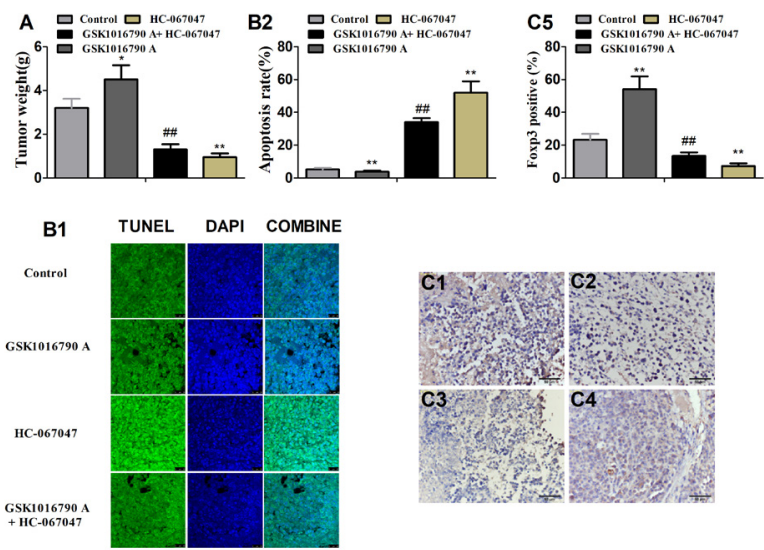

Figure 6. Inhibition of TRPV4 reduces A549 xenograft tumorigenicity in vivo.

(A) Tumour weight was measured at the end of study. (B1-B2) Apoptosis in tumour tissues was assessed by TUNEL staining, and images were captured at a final magnification of 200x. (C) Foxp3 expression in the implanted tumours was assessed by immunohistochemical staining. C1: control; C2: GSK1016790A (10 $\mu \mathrm{g} / \mathrm{kg} /$ day); C3: GSK1016790A (10 mg/kg/day) +HC-067047 (10 mg/kg/day); C4: $\mathrm{HC}-067047$ (10 mg/kg/day); C5: the number of Foxp3-positive cells in each image was semiquantitatively determined by ImageJ software. with the TRPV4 agonist GSK1016790A and reduced by treatment with the TRPV4 antagonist HC-067047 $(P<0.01)$, as evaluated by the final tumour weight and tissue apoptotic rates. Moreover, we found that HC067047 inhibited the GSK1016790A-induced increase in the development of tumours. Interestingly, with the control treatment, treatment with GSK1016790A caused higher expression of Foxp3 in the tumour tissue, while treatment with HC-067047 showed the opposite effect $(P<0.01)$.

\section{DISCUSSION}

In recent reports, TRPV4 was extensively studied due to its potential activities associated with human cancers. In some types of malignant tumours, TRPV4 exerts oncogenic effects. For instance, TRPV4 has been shown to promote the migration and invasion of glioma cells (Ou-Yang et al., 2018) and to promote metastasis through epithelial-mesenchymal transition in gastric cancer (Wang et al., 2020). Moreover, TRPV4 has been demonstrated to increase the malignant proliferation of tumour cells, such as oesophageal squamous cell carcinoma cells (Huang et al., 2019), hepatocellular carcinoma cells (Fang et al., 2018), and colon cancer cells (Liu et al., 2019). However, unexpectedly, TRPV4 was reported to exert antitumour effects in some types of tumours. Fusi and others (Fusi et al., 2014) found that TRPV4 is downregulated in keratinocytes in human nonmelanoma skin cancer tissues, and their research indicated that a selective reduction in TRPV4 expression could represent an early biomarker of skin carcinogenesis. Moreover, in another report, Peters and others (Peters et al., 2017) found that the pharmacological activation of TRPV4 by an agonist reduced the viability of basal breast cancer cell lines. However, the expression level and function of TRPV4 in NSCLC have not been reported. In the present study, we investigated the expression of TRPV4 in NSCLC patients and then focused on its role in the development of NSCLC. First, we performed immunohistochemical staining to investigate the expression level of TRPV4 in tumour tissues and para-carcinoma tissues. We found that the expression level of TRPV4 in tumour tissues was much higher than that in paralleled paracarcinoma tissues. Further study showed that TRPV4 expression was negatively correlated with the tumour size, but not other parameters, such as TNM stage and differentiation level, in NSCLC patients. Moreover, we assessed the expression of TRPV4 in NSCLC cell lines and in normal lung epithelial cells. Consistent with observations in clinical tumour tissues, the expression levels of TRPV4 in the A549, H1299 and H226 cells were significantly higher than those in the BEAS-2B cells. Previous studies (Baratchi et al., 2019; Xia et al., 2013) have shown that GSK1016790A and HC-067047 are an effective TRPV4 agonist and antagonist, in vivo and in vitro respectively, and have been widely used as tools to explore the function of TRPV4 under different conditions both in vivo and in vitro. Therefore, to obtain a better understanding of the mechanism of action of TRPV4 in NSCLC, we activated or inhibited TRPV4-mediated pathways by treatment with GSK1016790A and HC067047, respectively. As expected, we found that treatment with GSK1016790A could increase the proliferation of NSCLC cell lines in vitro and promote the growth of NSCLC cells in vivo, while HC-067047 led to the opposite results. Finally, we found that downregulation of TRPV4 by siRNA could promote NSCLC cell apoptotic 
death in vitro, while transfection of TRPV4 vectors produced opposite results. Together, these data demonstrate that TRPV4 promotes the growth of NSCLC.

In a previous investigations, TRPV4 was reported to target several intracellular proteins to promote the development of malignant tumours. For example, TRPV4 has been shown to regulate Akt phosphorylation (Ou-Yang et al., 2018) and then enhance glioma migration and invasion. In another study, TRPV4 was proven to activate the $\mathrm{Cdc} 42 / \mathrm{N}$-wasp pathway to promote glioblastoma invasion by altering cellular protrusions (Yang et al., 2020). Moreover, Fang and others (Fang et al., 2018) demonstrated that pharmacological inhibition of the TRPV4 channel suppresses the malignant biological behaviour of hepatocellular carcinoma via modulation of the ERK signalling pathway. To understand the molecular mechanism underlying the TRPV4-mediated pro-oncogenic activity, we analysed the relationship of TRPV4 with Foxp3 in the development of NSCLC. Foxp3 is a member of the forkhead/winged helix family of transcription factors. It is well known that Foxp3 is a specific transcription factor of Treg cells and plays an important role in regulating the function of Treg cells (Deng et al., 2019). Foxp3 is necessary to maintain the immunosuppressive effect of Treg cells (Göschl et al., 2019). However, it has been discovered that Foxp3 plays an important role in the progression of some types of malignant tumours. Foxp 3 has been reported to promote the development of cervical cancer and thyroid cancer (Luo et al., 2015; Chu et al., 2015). Additionally, an association of the FoxP3 SNP with a risk of NSCLC development was reported in the Chinese Han population (He et al., 2013), and NSCLC patients with high Foxp3 expression exhibit a significant decrease in overall survival and recurrence-free survival. A previous study demonstrated that Foxp3 promotes tumour growth and metastasis in NSCLC (Yang et al., 2017). Therefore, it is suggested that Foxp3 facilitates the EMT and promotes NSCLC growth and metastasis. TRPV1 has been reported to regulate the activator of transcription-3 (Lee et al., 2015), which is an important regulator of Foxp3 (Goldstein et al., 2016). Therefore, we hypothesized that TRPV4 may enhance cell proliferation and tumour growth by regulating the activation of Foxp3, which has never been reported. In the present study, we found that the TRPV4 level was closely associated with the Foxp3 level detected in the tumour tissues of NSCLC patients. We observed that the treatment of NSCLC cells with a TRPV4 antagonist or TRPV4 siRNA significantly reduced the expression of Foxp3, and similar results were observed in vivo. These results clearly indicated that TRPV4 could induce the expression of Foxp3, although we do not know whether it has a direct or indirect effect. To further verify our hypothesis, we investigated the effect of Foxp3 on TRPV4-mediated NSCLC proliferation in vitro. The results showed that downregulation of Foxp3 by siRNA transfection impaired TRPV4-induced NSCLC cell proliferation in vitro, supporting the idea that Foxp3 is required for TRPV4mediated tumour growth.

\section{CONCLUSION}

In summary, TRPV4 expression is upregulated in NSCLC. Inhibition of TRPV4 in NSCLC results in decreased growth and increased apoptosis, suggesting that TRPV4 plays a positive role in NSCLC development. Moreover, we provided evidence that the growth-stimulating effect of TRPV4 is related to Foxp3 overexpres- sion. Our results indicate that TRPV4 is a promising target for the treatment of NSCLC.

\section{Conflict of Interest Statement}

The authors declare that there are no conflicts of interest.

\section{REFERENCES}

Baratchi S, Keov P, Darby WG, Lai A, Khoshmanesh K, Thurgood P, Vahidi P, Ejendal K, McIntyre P (2019) The TRPV4 agonist GSK1016790A regulates the membrane expression of TRPV4 channels. Front Pharmacol 10: 6. https://doi.org/10.3389/fphar.2019.00006

Chen W, Zheng R, Baade PD, Zhang S, Zeng H, Bray F, Jemal A, Yu XQ, He J (2016) Cancer statistics in China, 2015. CA Cancer J Clin 66: 115-132. https://doi.org/10.3322/ caac.21338

Chu R, Liu SY, Vlantis AC, van Hasselt CA, Ng EK, Fan MD, Ng SK, Chan AB, Du J, Wei W, Liu X, Liu Z, Chen GG (2015) Inhibition of Foxp3 in cancer cells induces apoptosis of thyroid cancer cells. Mol Cell Endocrinol 399: 228-234. https://doi.org/10.1016/j. mce.2014.10.006

Deng G, Song X, Fujimoto S, Piccirillo CA, Nagai Y, Greene MI (2019) Foxp3 post-translational modifications and treg suppressive activity. Front Immunol 10: 2486. https://doi.org/10.3389/fimmu.2019.02486

Fang Y, Liu G, Xie C, Qian K, Lei X, Liu Q, Liu G, Cao Z, Fu J, Du H, Liu S, Huang S, Hu J, Xu X (2018) Pharmacological inhibition of TRPV4 channel suppresses malignant biological behavior of hepatocellular carcinoma via modulation of ERK signaling pathway. Biomed Pharmacother 101: 910-919. https://doi.org/10.1016/j. biopha.2018.03.014

Fusi C, Materazzi S, Minocci D, Maio V, Oranges T, Massi D, Nassini $R$ (2014) Transient receptor potential vanilloid 4 (TRPV4) is downregulated in keratinocytes in human non-melanoma skin cancer. $J$ Invest Dermatol 134: 2408-2417. https://doi.org/10.1038/jid.2014.145

Goldstein JD, Burlion A, Zaragoza B, Sendeyo K, Polansky JK, Huehn J, Piaggio E, Salomon BL, Marodon G (2016) Inhibition of the JAK/STAT signaling pathway in regulatory $\mathrm{T}$ cells reveals a very dynamic regulation of Foxp3 expression. PLoS One 11: e0153682. https://doi.org/10.1371/journal.pone.0153682

Göschl L, Scheinecker C, Bonelli M (2019) Treg cells in autoimmunity: from identification to Treg-based therapies. Semin Immunopathol 41: 301-314. https://doi.org/10.1007/s00281-019-00741-8

He YQ, Bo Q, Yong W, Qiu ZX, Li YL, Li WM (2013) FoxP3 genetic variants and risk of non-small cell lung cancer in the Chinese Han population. Gene 531: 422-425. https://doi.org/10.1016/j. gene.2013.08.066

Huang R, Wang F, Yang Y, Ma W, Lin Z, Cheng N, Long Y, Deng S, Li Z (2019) Recurrent activations of transient receptor potential vanilloid-1 and vanilloid-4 promote cellular proliferation and migration in esophageal squamous cell carcinoma cells. FEBS Open Bio 9: 206-225. https://doi.org/10.1002/2211-5463.12570

Liu X, Zhang P, Xie C, Sham KWY, Ng SSM, Chen Y, Cheng CHK (2019) Activation of PTEN by inhibition of TRPV4 suppresses colon cancer development. Cell Death Dis 10: 460. https://doi. org/10.1038/s41419-019-1700-4

Lee E, Jung DY, Kim JH, Patel PR, Hu X, Lee Y, Azuma Y, Wang HF, Tsitsilianos N, Shafiq U, Kwon JY, Lee HJ, Lee KW, Kim JK (2015) Transient receptor potential vanilloid type-1 channel regulates diet-induced obesity, insulin resistance, and leptin resistance. FASEB J 29: 3182-3192. https://doi.org/10.1096/fj.14-268300

Luo M, Mo J, Yu Q, Zhou S, Ning R, Zhang Y, Su C, Wang H, Cui J (2018) Wogonoside induces apoptosis in human non-small cell lung cancer A549 cells by promoting mitochondria dysfunction. Biomed Pharmacother 106: 593-598. https://doi.org/10.1016/j. biopha.2018.06.077

Luo Q, Zhang S, Wei H, Pang X, Zhang H (2015) Roles of Foxp3 in the occurrence and development of cervical cancer. Int J Clin Exp Pathol 8: 8717-830

Ou-Yang Q, Li B, Xu M, Liang H (2018) TRPV4 promotes the migration and invasion of glioma cells via AKT/Rac1 signaling. Biochem Biophys Res Commun 503: 876-881. https://doi.org/10.1016/j. bbrc.2018.06.090

Peters AA, Jamaludin SYN, Yapa KTDS, Chalmers S, Wiegmans AP, Lim HF, Milevskiy MJG, Azimi I, Davis FM, Northwood KS, Pera E, Marcial DL, Dray E, Waterhouse NJ, Cabot PJ, Gonda TJ, Kenny PA, Brown MA, Khanna KK, Roberts-Thomson SJ, Monteith GR (2017) Oncosis and apoptosis induction by activation of an overexpressed ion channel in breast cancer cells. Oncogene 36: 6490-6500. https://doi.org/10.1038/onc.2017.234

Santoni G, Farfariello V (2011) TRP channels and cancer: new targets for diagnosis and chemotherapy. Endocr Metab Immune Disord Drug Targets 11: 54-67. https://doi.org/10.2174/187153011794982068 
Santoni G, Farfariello V, Amantini C (2011) TRPV channels in tumor growth and progression. Adv Exp Med Biol 704: 947-967. https:// doi.org/10.1007/978-94-007-0265-3_49

Siegel RL, Miller KD, Jemal A (2017) Cancer Statistics, 2017. CA Cancer I Clin 67: 7-30. https://doi.org/10.3322/caac.21387

Siegel RL, Miller KD, Jemal A (2018) Cancer statistics, 2018. CA Cancer I Clin 68: 7-30. https://doi.org/10.3322/caac.21442

Travis WD, Brambilla E, Nicholson AG, Yatabe Y, Austin JHM, Beasley MB, Chirieac LR, Dacic S, Duhig E, Flieder DB, Geisinger K, Hirsch FR, Ishikawa Y, Kerr KM, Noguchi M, Pelosi G, Powell CA, Tsao MS, Wistuba I; WHO Panel (2004) The 2015 World Health Organization classification of lung tumors: impact of genetic, clinical and radiologic advances since the 2004 classification. J Thorac Oncol 10: 1243-1260. https://doi.org/10.1097/ JTO.0000000000000630

Wang H, Zhang B, Wang X, Mao J, Li W, Sun Y, Yuan Y, Ben Q, Hua L, Qian A (2020) TRPV4 Overexpression promotes metastasis through epithelial-mesenchymal transition in gastric cancer and correlates with poor prognosis. Onco Targets Ther 13: 8383-8394. https://doi.org/10.2147/O'T'T.S256918

Xia Y, Fu Z, Hu J, Huang C, Paudel O, Cai S, Liedtke W, Sham JS (2013) TRPV4 channel contributes to serotonin-induced pulmonary vasoconstriction and the enhanced vascular reactivity in chronic hypoxic pulmonary hypertension. Am J Physiol Cell Physiol 305: C704C715. https://doi.org/10.1152/ajpcell.00099.2013

Yang S, Liu Y, Li MY, Ng CSH, Yang SL, Wang S, Zou C, Dong Y, Du J, Long X, Liu LZ, Wan IYP, Mok T, Underwood MJ, Chen GG (2017) FOXP3 promotes tumor growth and metastasis by activating Wnt/B-catenin signaling pathway and EMT in non-small cell lung cancer. Mol Cancer 16: 124. https://doi.org/10.1186/s12943017-0700-1

Yang W, Wu PF, Ma JX, Liao MJ, Xu LS, Yi L (2020) TRPV4 activates the $\mathrm{Cdc} 42 / \mathrm{N}$-wasp pathway to promote glioblastoma invasion by altering cellular protrusions. Sci Rep 10: 14151. https://doi. org/10.1038/s41598-020-70822-4 